ISSN 0103-8478

\title{
Dietas larvais alternativas para criação massal da mosca da bicheira, Cochliomyia hominivorax
}

\author{
Alternative larval diets for mass rearing of screwworm, Cochliomyia hominivorax
}

\author{
Thiago Mastrangelo ${ }^{\mathrm{I}}$ Fernando Bezerra $^{\mathrm{II}}$ Thiago Fernandes ${ }^{\mathrm{II}}$
}

\section{RESUMO}

A criação massal da mosca da bicheira, Cochliomyia hominivorax (Diptera: Calliphoridae), para programas que utilizam a Técnica do Inseto Estéril nas Américas faz parte de um dos maiores programas do mundo de criação de insetos em larga escala. A maior parte do custo e do trabalho necessários para a produção massal está relacionada com a dieta larval, o que torna importante o seu aprimoramento levando-se em conta sua eficiência, custo e praticidade. Neste trabalho, foram realizados dois bioensaios com dietas alternativas feitas a partir de produtos disponíveis no mercado brasileiro com o objetivo de avaliar a viabilidade e eficiência dessas dietas para criações de mosca da bicheira no Brasil. Foram testadas duas dietas líquidas (tendo como bulking agents bagaço de cana ou fibra de coco), uma de carne, e outra de gel. Os parâmetros biológicos (peso das larvas, viabilidade larval, peso de pupas, porcentagem de emergência de adultos e razão sexual) estiveram, em todos os casos, dentro de parâmetros satisfatórios para a criação laboratorial de $\boldsymbol{C}$. hominivorax e as dietas não diferiram estatisticamente entre si. Devido a vantagens importantes, como alta viabilidade larval e menor custo, recomenda-se a dieta de gel para as criações de larvas da mosca da bicheira no Brasil.

Palavras-chave: larva, biofábrica, Cochliomyia hominivorax.

\section{ABSTRACT}

The mass rearing of the screwworm fly, Cochliomyia hominivorax (Diptera: Calliphoridae), for programs that integrate the Sterile Insect Technique in the Americas is part of one of the largest insect mass rearing programs in the world. Most of the cost and labor required in mass rearing systems is related to the larval diet, what enhances the relevance of the constant development of novel diets taking into account their effectiveness, cost and practicability. In this research, two bioassays with alternative diets made using local products were performed in order to evaluate the feasibility and efficiency of these diets for screwworm rearing in Brazil. Two liquid diets (having sugarcane bagasse or coconut fiber as bulking agents), a meat diet, and a gelled diet were tested. The biological parameters evaluated (larval weight, larval viability, pupal weight, adult emergence, and sex ratio) were satisfactory according to mass rearing standards and the diets did not differ statistically $(P>0.05)$. Due to its higher number of advantages, as high larval viability and lower cost, the gelled diet developed is recommended for screwworm larvae rearing in Brazil.

Key words: larvae, biofactory, Cochliomyia hominivorax.

\section{INTRODUÇÃO}

Um dos maiores projetos em vigência no mundo para erradicação de insetos pelo uso da Técnica do Inseto Estéril envolve a criação massal da mosca da bicheira, Cochliomyia hominivorax (Coquerel), sob responsabilidade da Comisión México-Americana para la Erradicación del Gusano Barrenador del Ganado (COMEXA) e da Comisión Panamá-Estados Unidos para la Erradicación y Prevención del Gusano Barredor del Ganado (COPEG) (MASTRANGELO et al., 2012). C. hominivorax foi erradicada dos E.U.A. e da América Central através de um programa de erradicação em área-ampla integrando a Técnica do Inseto Estéril (VARGAS-TERÁN et al., 2005). Os objetivos das atuais campanhas de erradicação são prevenir reinfestações das áreas livres, eliminar essa praga das ilhas do Caribe, e fomentar a criação de novas áreas livres em países da America do Sul. A biofábrica

\footnotetext{
IUniversidade Estadual de Campinas (UNICAMP), 13083-875, Campinas, SP, Brasil. E-mail: mastrangelo@cbmeg.unicamp.br. Autor para correspondência.

IIUniversidade Federal do Vale do São Francisco (UNIVASF), Petrolina, PE, Brasil.

IIIUniversidade Metodista de Piracicaba (UNIMEP), Piracicaba, SP, Brasil.
} 
de moscas estéreis da COPEG localizada em Pacora, Panamá, produz atualmente cerca de 40 milhões de machos estéreis por semana para suprir a barreira de insetos estéreis estabelecida na fronteira entre Panamá e Colômbia, prevenindo a entrada de moscas férteis vindas do norte da Colômbia. Aproximadamente 20 toneladas de ingredientes de dieta (a um custo de quase US\$ 30.000 .semana ${ }^{-1}$ ) são necessárias para manter essa produção (CHAUDHURY \& SKODA, 2007).

A maior parte do custo e do trabalho necessários para a produção massal da C.hominivorax estão relacionados com a dieta larval. Mais de 15.000 L de dieta larval, como exemplo, são preparados diariamente na biofábrica de COPEG (CHAUDHURY \& SKODA, 2007; MASTRANGELO et al., 2012).

A pesquisa para o desenvolvimento de novas dietas larvais é importante, pois além de afetarem o desempenho dos estágios imaturos do inseto (CANGUSSU \& ZUCOLOTO, 1997), podem ter um grande impacto no custo total da produção massal (CHAUDHURY \& SKODA, 2007). Estudos sobre a nutrição de $C$. hominivorax são realizados desde a década de 1930 (MELVIN \& BUSHLAND, 1936; GRAHAM \& DUDLEY, 1959; BROWN \& SNOW, 1979; HARRIS et al., 1984, 1985; TAYLOR \& MANGAN, 1987; TAYLOR, 1988; TAYLOR et al., 1991; CHAUDHURY \& ALVAREZ, 1999; CHAUDHURY et al., 2002; CHAUDHURY et al., 2011) e uma das razões para o sucesso das campanhas de erradicação dessa praga realizadas anteriormente foi o esforço contínuo de melhoramento das técnicas de criação dela.

Apesar da existência de diversos tipos de dietas para larvas de C. hominivorax no exterior, ainda hoje poucos pesquisadores do Brasil as utilizam, principalmente pela dificuldade de importação e alto custo de certos ingredientes. Por isso, ainda são empregadas quase exclusivamente dietas a base de carne moída e sangue (SILVA et al., 2008; RABÊLO et al., 2011), as quais são desprovidas de uniformidade de qualidade e apresentam baixa durabilidade. Neste trabalho, foram realizados dois bioensaios com quatro dietas alternativas feitas a partir de produtos de origem brasileira para se avaliar a sua viabilidade e eficiência para a produção laboratorial de larvas da mosca da bicheira no Brasil.

\section{MATERIAL E MÉTODOS}

Foram testados separadamente dois grupos de dietas para larvas de $\boldsymbol{C}$. hominivorax. No primeiro grupo, foram testadas duas dietas liquidas:

a) Dieta líquida + bagaço de cana

b) Dieta líquida + fibra de coco
Num segundo grupo, foram testadas uma dieta a base de carne e outra com um agente gelificante:

c) Dieta larval de iniciação

d) Dieta de gel

As dietas foram baseadas em GRAHAM \& DUDLEY (1959), TAYLOR et al. (1991) e CHAUDHURY \& SKODA (2007). As dietas líquidas do $1^{\circ}$ grupo continham como formulação básica $60 \mathrm{~g}$ de hemácias spray dried (AP301 ${ }^{\circledR}$ - American Protein Corp., Araçatuba, SP), $40 \mathrm{~g}$ de ovo spray dried (Sohovos - AB Brasil Ind. e Com. de Alimentos, Ltda), $40 \mathrm{~g}$ de leite em pó desnatado, $1 \mathrm{~g}$ de ácido cítrico, $1 \mathrm{~g}$ de citrato de sódio, $1 \mathrm{~mL}$ de formaldeído e $800 \mathrm{~mL}$ de água. Foram testados como bulking agents: a) bagaço de cana, ou b) fibra de coco $(2,7$ $\mathrm{g}$ de cada para $700 \mathrm{~mL}$ de dieta líquida). O bagaço de cana moído (seco e peneirado) foi obtido da Usina AGROVALE e a fibra de coco foi adquirida como substrato comercial $\left(\mathrm{SOCOCO}^{\circledR}\right)$ para orquídeas (pacotes com $15 \mathrm{~L}$ de fibra de coco verde desfibrado, lavado, e sem pó).

No segundo grupo, a dieta a base de carne seguia a mesma formulação daquela utilizada como dieta larval de iniciação da colônia-mãe (para 1 bandeja de $3 \mathrm{~L}$ : $328 \mathrm{~g}$ de carne crua bovina de $1^{\mathrm{a}}$ moída para $350 \mathrm{~mL}$ de dieta líquida, a qual era preparada a partir de $21 \mathrm{~g}$ de hemácias spray dried, $14 \mathrm{~g}$ de ovo spray dried, $14 \mathrm{~g}$ de leite em pó desnatado, $350 \mathrm{mg}$ de ácido cítrico, $350 \mathrm{mg}$ de citrato de sódio, $0,35 \mathrm{~mL}$ de formaldeído e $300 \mathrm{~mL}$ de água).

Para o preparo de $1 \mathrm{~L}$ da dieta de gel, foi utilizada a formulação a seguir: $60 \mathrm{~g} \mathrm{de}$ hemácias spray dried, $40 \mathrm{~g}$ de ovo spray dried, $40 \mathrm{~g}$ de leite em pó desnatado, $30 \mathrm{~g}$ de Ecogel $^{\circledR}, 1$ $\mathrm{mL}$ de formaldeído e $800 \mathrm{~mL}$ de água. O Ecogel ${ }^{\circledR}$ é feito a partir de polímeros como poliacrilato de poliacrilamida, é comercializado como condicionador de solo, e, na proporção correta (obtida durante os pré-testes e utilizada neste trabalho), proporciona textura e viscosidade ideais para a locomoção e alimentação das larvas.

As dietas $(700 \mathrm{~mL})$ foram acondicionadas em potes plásticos $(15 \times 10 \times 5 \mathrm{~cm})$, com tampa telada com tecido voile para permitir aeração, e mantidas em uma torre aquecida a $30-36^{\circ} \mathrm{C}$ sob luz ambiente e umidade relativa entre $60-90 \%$ por até 5 dias. As massas de ovos ou larvas utilizadas para os testes foram provenientes da colônia-mãe de $\boldsymbol{C}$. hominivorax, cujas condições de criação foram descritas por MASTRANGELO et al. (2012).

$\mathrm{O}$ teste com as dietas líquidas teve que ser realizado com larvas de $2^{\circ}$ instar, porque a viabilidade 
das larvas de $1^{\circ}$ instar, depois que as massas de ovos eram colocadas diretamente sobre o bagaço de cana ou fibra de coco, foi muito baixa e variável (entre 0 e $25 \%$ somente). Portanto, 100 larvas de $2^{\circ}$ instar foram retiradas de uma dieta padrão de iniciação e colocadas em cada parcela experimental.

No teste com as dietas a base de carne e gel, uma massa de $5 \mathrm{mg}$ de ovos ( $\approx 100$ ovos) foi colocada sobre um pedaço $(3 \times 3 \mathrm{~cm})$ de papel toalha neutro umedecido sobre a dieta de cada parcela. Em geral, na criação de $\boldsymbol{C}$. hominivorax, a dieta larval deve ser adicionada aos poucos ao longo do período larval, caso contrário, as larvas não conseguem completar o desenvolvimento por causa do apodrecimento, contaminação e ressecamento (BAUMHOVER et al. 1966; CHAUDHURY \& SKODA, 2007; CHAUDHURY \& SKODA, 2009). Portanto, com relação à frequência de adição das dietas, as dietas líquidas tiveram que ser adicionadas gradativamente, duas vezes ao dia, por 4 dias consecutivos, enquanto que, para a dieta a base de carne e de gel, foram adicionados $25 \%$ da dieta no $1^{\circ}$ dia, $50 \%$ no $3^{\circ}$ dia, e $25 \%$ no $4^{\circ}$ dia, completando o volume de $700 \mathrm{~mL}$.

Quando as larvas começavam a escalar as paredes dos potes para tentar pupar $\left(5^{\circ}\right.$ dia após a eclosão das larvas ou final do $3^{\circ}$ instar), os potes foram colocados sobre bandeja $(50 \times 30 \times 10 \mathrm{~cm})$ com vermiculita (expandida tamanho médio) e abertos para permitir a pupação. Ao final dos testes, o descarte das dietas foi colocado em sacolas plásticas e armazenado a $-20^{\circ} \mathrm{C}$.

Os tratamentos foram dispostos em delineamento inteiramente casualizado, havendo 3 repetições por tratamento. Os parâmetros avaliados nos dois testes foram: peso das larvas de $3^{\circ}$ instar (ao abandonarem a dieta), peso de pupas $\left(5^{\circ}\right.$ dia após a pupação), viabilidade larval (definida como: $n^{o}$ de pupas $\div n^{o}$ inicial de larvas, sendo larvas de $2^{\circ}$ instar, no teste com dietas líquidas, e de $1^{\circ}$, no teste com dietas de gel ou carne), porcentagem de emergência de adultos (de amostras com 100 pupas em placas de Petri), e razão sexual ( $n^{0}$ de fêmeas/ $\mathrm{n}^{\mathrm{o}}$ de machos+fêmeas). As médias dos parâmetros biológicos foram comparadas pelo teste-t de Student $(\alpha=0,05)$. As análises foram efetuadas através do programa estatístico SAS 9.1 (SAS Institute, 2003).

\section{RESULTADOS E DISCUSSÃO}

Os valores de peso e viabilidade larvais, peso pupal (pupas com 5 dias de idade), emergência de adultos e razão sexual obtidos das dietas dos dois testes (Tabelas 1 e 2) estiveram todos dentro de parâmetros satisfatórios para criação de $\boldsymbol{C}$. hominivorax em laboratório, embora não tenham sido detectadas diferenças significativas $(\mathrm{P}>$ $0,05)$. Também não foi observada diferença no tempo de desenvolvimento larval entre as dietas, ficando sempre em torno de 4-5 dias.

O peso das larvas de $3^{\circ}$ instar foi de $52,7 \pm 6,4 \mathrm{mg}$ na dieta líquida com bagaço de cana, $55,3 \pm 4,4 \mathrm{mg}$ na dieta líquida com fibra de coco, $47,7 \pm 2,6 \mathrm{mg}$ na dieta a base de carne e $58,7 \pm 4,7$ $\mathrm{mg}$ na dieta de gel. TAYLOR \& MANGAN (1987) relataram valores de peso larval entre 40 e $80 \mathrm{mg}$ para testes com dietas a base de carne e gel. CHAUDHURY \& SKODA (2007) obtiveram valores entre 47 e $74 \mathrm{mg}$ em testes de dietas de gel e a base de fibra de celulose.

O peso pupal em todas as dietas dos dois testes também foi elevado, sendo sempre maior ou igual a $47 \mathrm{mg}$. Segundo VREYSEN et al. (2007), o peso mínimo aceitável para pupas irradiadas com cinco dias de idade destinadas à liberação em campo, deve ser de $47 \mathrm{mg}$.

Duas grandes desvantagens das dietas líquidas com bagaço de cana ou fibra de coco foram a necessidade de se utilizar larvas de $2^{\circ}$ instar e a maior frequência de adição de dieta diariamente. Na verdade, há poucos

Tabela 1 - Parâmetros biológicos (média \pm erro padrão) de Cochliomyia hominivorax em diferentes dietas líquidas para larvas.

\begin{tabular}{lcccc}
\hline Dieta & Peso larval (mg) & Viabilidade larval (\%) & Peso pupal (mg) & Emergência (\%) $^{\text {Razão sexual }}$ \\
\hline Bagaço de cana & $52,7 \pm 6,4 \mathrm{a}^{\mathrm{a}}$ & $96,7 \pm 2,3 \mathrm{a}$ & $55,3 \pm 3,7 \mathrm{a}$ & $95 \pm 0,9 \mathrm{a}$ \\
Fibra de coco & $55,3 \pm 4,4 \mathrm{a}$ & $98 \pm 0,58 \mathrm{a}$ & $50,7 \pm 3,2 \mathrm{a}$ & $85 \pm 4,8 \mathrm{a}$ \\
& & & \\
& $F_{1,5}=0,12$ & $F_{1,5}=0,31$ & $F_{1,5}=0,91$ & $F_{1,5}=4,2$ \\
ANOVA (LSD) & $P=0,74$ & $P=0,61$ & $P=0,39$ & $P=0,112 \mathrm{a}$ \\
& C.V. ${ }^{\mathrm{c}}=17,5 \%$ & C.V. $=3,02 \%$ & C.V. $=11,3 \%$ & $F_{1,5}=0,03$ \\
C.V. $=6,7 \%$ & $P=0,87$ \\
\hline
\end{tabular}

\footnotetext{
${ }^{a}$ Médias na mesma coluna seguidas da mesma letra não diferem significativamente entre si pelo teste-t de Student $(P>0,05)$.

${ }^{\mathrm{b}}$ Razão sexual $=\mathrm{n}^{\mathrm{o}}$ de fêmeas $/\left(\mathrm{n}^{\mathrm{o}}\right.$ de machos $+\mathrm{n}^{\mathrm{o}}$ de fêmeas $)$.

${ }^{\mathrm{c}} \mathrm{C} . \mathrm{V} .=$ coeficiente de variação.
} 
Tabela 2 - Parâmetros biológicos (média \pm erro padrão) de Cochliomyia hominivorax em diferentes dietas larvais.

\begin{tabular}{|c|c|c|c|c|c|}
\hline Dieta & Peso larval (mg) & Viabilidade larval (\%) & Peso pupal (mg) & Emergência (\%) & Razão sexual $^{\text {b }}$ \\
\hline Carne & $47,7 \pm 2,6 \mathrm{a}^{\mathrm{a}}$ & $89,3 \pm 5,2 \mathrm{a}$ & $53 \pm 1,7 \mathrm{a}$ & $92,7 \pm 1,9 \mathrm{a}$ & $0,47 \pm 0,06 \mathrm{a}$ \\
\hline Gel & $58,7 \pm 4,7 \mathrm{a}$ & $93 \pm 5,1 \mathrm{a}$ & $52 \pm 2,52 \mathrm{a}$ & $80 \pm 6,2 \mathrm{a}$ & $0,56 \pm 0,03 \mathrm{a}$ \\
\hline ANOVA (LSD) & $\begin{array}{l}F_{1,5}=4,2 \\
P=0,11 \\
\text { C.V. }{ }^{c}=12,4 \%\end{array}$ & $\begin{array}{l}F_{1,5}=0,26 \\
P=0,64 \\
\text { C.V. }=9,7 \%\end{array}$ & $\begin{array}{l}F_{1,5}=0,11 \\
P=0,76 \\
\text { C.V. }=7,1 \%\end{array}$ & $\begin{array}{l}F_{1,9}=3,8 \\
P=0,09 \\
\text { C.V. }=11,9 \%\end{array}$ & $\begin{array}{l}F_{1,9}=1,6 \\
P=0,25 \\
\text { C.V. }=19,6 \%\end{array}$ \\
\hline
\end{tabular}

${ }^{a}$ Médias na mesma coluna seguidas da mesma letra não diferem significativamente entre si pelo teste-t de Student $(P>0,05)$.

${ }^{\mathrm{b}}$ Razão sexual $=\mathrm{n}^{\mathrm{o}}$ de fêmeas $/\left(\mathrm{n}^{\mathrm{o}}\right.$ de machos $+\mathrm{n}^{\mathrm{o}}$ de fêmeas $)$.

${ }^{\mathrm{c}} \mathrm{C} . \mathrm{V} .=$ coeficiente de variação.

relatos na literatura de bioensaios com dietas líquidas para criação de $\boldsymbol{C}$. hominivorax. BAUMHOVER et al. (1966) relataram a tentativa de utilização de uma dieta semilíquida na biofábrica em Mission, Texas. A dieta era a base de carne moída, plasma bovino, água e formalina, e eram colocadas camadas de algodão que flutuavam na bandeja, dando suporte para as larvas e evitando afogamento. Dessa forma, a densidade larval podia ser aumentada porque as larvas se dispersavam mais na bandeja ao invés de se alimentarem com o comportamento de "ondas", como nas outras dietas.

A dieta com Ecogel ${ }^{\circledR}$ proporcionou alta viabilidade larval e não houve diferença significativa entre os pesos de larvas de $3^{\circ}$ instar em comparação com dieta a base de carne (Tabela 2). Embora o período larval tenha sido praticamente o mesmo nas duas dietas (4-5 dias), mais de 90\% das prépupas da dieta a base de carne abandonou o meio em menos de $15 \mathrm{~h}$ a partir do início da pupação (provavelmente para escapar do excesso de resíduos tóxicos e da carne em putrefação) (TAYLOR \& MANGAN, 1987; CHAUDHURY \& SKODA, 2009), enquanto que foi possível encontrar larvas de $3^{\circ}$ instar se alimentando na dieta de gel. O Ecogel ${ }^{\circledR}$ também serviu, aparentemente, para absorver os resíduos metabólicos excretados pelas larvas, o que reduziu consideravelmente o mau cheiro (odores característicos de amônia e enxofre) emitido pela dieta. Além disso, a dieta de gel manteve-se semisólida todo o tempo, sem aumento da viscosidade (o que atrapalharia a saída das larvas), mas podia ser dissolvida rapidamente em água, facilitando, assim, seu descarte após congelamento por $24 \mathrm{~h}$ em freezer.

Outra grande vantagem da dieta de gel foi o seu menor custo. Para produzir 100 mLde pupas $(\approx 830$ pupas $)$, o custo da dieta a base de carne (500 g de carne moída $+300 \mathrm{~mL}$ de dieta líquida) foi de $\mathrm{R} \$ 6,60$, enquanto a de gel $(\approx 1 \mathrm{~L})$ foi de apenas $\mathrm{R} \$ 1,89$ (economia de quase $72 \%$ ou 3,5 vezes).
Trabalhos anteriores demonstraram a viabilidade de dietas de gel para a mosca da bicheira. A dieta a base de carne desenvolvida por MELVIN \& BUSHLAND (1936) foi utilizada praticamente sem alterações para a produção massal de $\boldsymbol{C}$. hominivorax até 1970. GINGRICH et al. (1971) desenvolveram uma dieta líquida, depois modificada por BROWN \& SNOW (1979), composta de sangue e ovo spray dried, leite em pó, formalina e água, além de algodão ou fibras de acetato para dar sustentação às larvas, sendo utilizada nos programas de erradicação de $\boldsymbol{C}$. hominivorax por mais de 12 anos. HARRIS et al. (1984) testaram vários agentes gelificantes para a dieta de GINGRICH et al. (1971) e relataram que quatro deles (Gelgard, carragenina, caseína e goma Karaya) permitiam uma produção de larvas com qualidade semelhante às da dieta de carne. HARRIS et al. (1985) demonstraram que o agente gelificante de poliacrilato de poliacrilamida de sódio (WaterLock G-400) era promissor para a formulação de dietas larvais. TAYLOR \& MANGAN (1987) então compararam os parâmetros de controle de qualidade de moscas criadas na dieta de carne padrão e na de gel Water-Lock ao longo de cinco gerações e não encontraram diferenças significativas.

No ano seguinte, TAYLOR(1988) comparou as dietas a base de Water-Lock (70 $\mathrm{g}$ de sangue spray dried, $30 \mathrm{~g}$ de ovo spray dried, $30 \mathrm{~g}$ de leite em pó, $1,2 \mathrm{~mL}$ de formalina, $12 \mathrm{~g}$ de Water-Lock G-400 e 1 L de água) e carragenina para produção de larvas em pequena escala e massal. A dieta com Water-Lock foi superior tanto na iniciação larval quanto na criação. Apesar disso, a biofábrica do COMEXA adotou, em agosto de 1986, a dieta a base de carragenina $(6 \%$ de sangue spray dried, $3 \%$ de ovo spray dried, $3 \%$ de leite em pó, $0,1 \%$ de formalina, $0,25 \%$ de carragenina e $87,65 \%$ de água) para a iniciação larval.

Posteriormente, TAYLOR et al. (1991) compararam os sistemas de produção baseados na 
dieta de gel Water-Lock e o padrão (GINGRICH et al., 1971). O sistema com dieta de gel rendeu $2 \%$ de pupas com maior peso, viabilidade ovo-pupa $32 \%$ maior e exigiu menos $54 \%$ de dieta e menos $88 \%$ de trabalho do que o sistema padrão. Depois desse trabalho, a conversão completa da biofábrica do COMEXA para o sistema de criação em dieta de gel Water-Lock começou em outubro de 1989 e foi concluída em abril de 1990. Até recentemente, a dieta larval utilizada na biofábrica do COMEXA era praticamente a mesma (60 $\mathrm{g}$ de sangue spray dried, $40 \mathrm{~g}$ de ovo spray dried, $40 \mathrm{~g}$ de leite em pó, $1 \mathrm{~mL}$ de formalina, $18 \mathrm{~g}$ de Aquatain e $841 \mathrm{~mL}$ de água) (CHAUDHURY \& SKODA, 2007).

Agentes gelificantes como o Water-Lock ou o Ecogel ${ }^{\circledR}$ possuem inúmeras vantagens como mostraram os trabalhos citados. A dieta permanece semi-sólida ao longo do tempo, emite menos odores de enxofre e amônia, e mantém a qualidade nutricional por mais tempo para as larvas, antes de terem seu desenvolvimento prejudicado pela contaminação com os resíduos tóxicos. Por causa da pouca disponibilidade de água livre, os resíduos metabólicos solúveis, como amônia, não se difundem para as partes ainda não aproveitadas pelas larvas, eliminando a necessidade de se remover a dieta podre e reduzindo a frequência de adição de dieta (CHAUDHURY \& SKODA, 2007). $\mathrm{O}$ agente gelificante também não exige aquecimento durante o preparo da dieta, como ágar ou carragenina, podendo ser utilizado imediatamente.

Devido ao maior número de vantagens, em comparação às outras dietas, a dieta de gel testada neste trabalho está sendo utilizada no Centro de Biologia Molecular e Engenharia Genética da UNICAMP tanto para a manutenção das criações de C. hominivorax, como também das moscas varejeiras Cochliomyia macellaria, Chrysomya albiceps e Chrysomya megacephala. A única mudança na formulação foi a adoção de outro agente gelificante, o FORTH Gel ${ }^{\circledR}$, o qual está sendo utilizado na proporção de $22 \mathrm{~g}$ para se fazer $1 \mathrm{~L}$ da dieta de gel. Depois de 11 gerações, os parâmetros biológicos para as quatro espécies vêem se mantendo estáveis (Mastrangelo, dados não publicados).

\section{CONCLUSÃO}

A criação de larvas de Cochliomyia hominivorax em dietas líquidas ou de gel é viável. A dieta a base de gel testada não apresentou diferença em relação à dieta a base de carne moída quanto ao desenvolvimento das larvas, apresentando vantagens técnicas e econômicas que justificam sua utilização em biofábricas.

\section{AGRADECIMENTOS}

À Fundação de Amparo à Pesquisa do Estado de São Paulo (FAPESP) pela concessão da bolsa de Doutorado e ao Conselho Nacional de Desenvolvimento Científico e Tecnológico (CNPq) pela concessão das bolsas de iniciação científica.

\section{REFERÊNCIAS}

BAUMHOVER, A.H.; HUSMAN, C.N.; GRAHAM, A.J. Screwworms. In: SMITH, C.N. (Ed.). Insect colonization and mass production. New York: Academic Press, 1966. p. 533-554.

BROWN, H.E.; SNOW, J.W. Screwworms (Diptera: Calliphoridae): A new liquid media for rearing screwworm larvae. Journal of Medical Entomology, Lanham, v. 16, p. 29-32, 1979. Disponível em: <http://www.ingentaconnect.com/content/esa/ jme/1979/00000016/00000001/art00004?crawler=true $>$. Acesso em: 20 jan. 2013.

CANGUSSU, J.A.; ZUCOLOTO, F.S. Effect of protein sources on fecundity, food acceptance, and sexual choice by Ceratitis capitata (Diptera, Tephritidae). Revista Brasileira de Biologia, Rio de Janeiro, v. 57, p. 611-618, 1997. Disponível em: <http://cppmet.ufpel. edu.br/cppmet/index3.php $>$ ?secao=0. Acesso em: 15 jan. 2013.

CHAUDHURY, M.F.; ALVAREZ, L.A. A new starchgrafted gelling agent for screwworm (Diptera: Calliphoridae) larval diet. Journal of Economic Entomology, College Park, v. 92, p. 1138-1141, 1999. Disponível em: <http://www.ingentaconnect. com/content/esa/jee/1999/00000092/00000005/art00020\#expand/ collapse> Acesso em: 20 jan. 2013.

CHAUDHURY, M.F.; SKODA, S.R. A cellulose fiber-based diet for screwworm (Diptera: Calliphoridae) larvae. Journal of Economic Entomology, College Park, v. 100, n. 1, p. 241-245, 2007. Disponível em: <http://digitalcommons.unl.edu/cgi/viewcontent.cg i? article $=1792 \&$ context $=$ usdaarsfacpub $>$. Acesso em: 18 jan. 2013.

CHAUDHURY, M.F.; ALVAREZ, L.A.; VELAZQUEZ, L.L. Recycled paper products as substitutes for gelling agent in screwworm (Diptera: Calliphoridae) larval diet. Journal of Economic Entomology, College Park, v. 95, p. 13371341, 2002. Disponível em: <http://www.ncbi.nlm.nih.gov/ pubmed/12539852>. Acesso em: 18 jan. 2013.

CHAUDHURY, M.F.; SKODA, S.R. Diet pH, and viscosity affect development and survival of screwworm larvae (Diptera: Calliphoridae). Journal of Economic Entomology. College Park v.102, p. 799-803, 2009. Disponível em: <http://www.ars.usda.gov/ research/publications/publications.htm?SEQ NO $115=231435>$. Acesso em: 20 jan. 2013.

CHAUDHURY, M.F.; SKODA, S.R.; SAGEL, A. Solidifying agent and processing of blood used for the larval diet affect screwworm (Diptera: Calliphoridae) life-history parameters. Journal of Economic Entomology, College Park, v. 103, p. 11031107, 2011. Disponível em: <http://dx.doi.org/10.1603/EC10375>. Acesso em: 20 jan. 2013.

GRAHAM, A.J.; DUDLEY, F.H. Culture methods for mass rearing of screw-worm larvae. Journal of Economic Entomology, College Park, v. 52, p. 1006-1008, 1959. Disponível em: <http://www. ingentaconnect.com/content/esa/jee/1959/00000052/00000005/ art00064>. Acesso em: 20 jan. 2013. 
GINGRICH, R.E.; GRAHAM, A.J.; HIGHTOWER, B.G. Media containing liquefied nutrients for mass rearing larvae of the screwworm. Journal of Economic Entomology, College Park, v. 64, p. 678-683, 1971. Disponível em: <http://www.ncbi.nlm.nih.gov/ pubmed/5558276>. Acesso em: 20 jan. 2013.

HARRIS, R.L.; PETERSON, R.D.; VASQUEZ-G., M.E.; GRAHAM, O.H. Gelled media for the production screwworm larvae. Southwestern Entomologist, Dallas, v. 9, p. 257-262, 1984. Disponível em: <http://sswe.tamu.edu/articles/PDF/SWE_ V14 N1 P067-70.pdf>. Acesso em: 20 jan. 2013.

HARRIS, R.L.; GERSABECK, E.F.; CORSO, C.; GRAHAM, O.H. Screwworm larval production on gelled media. Southwestern Entomologist, Dallas, v. 10, p. 253-256, 1985. Disponível em: <http://agris.fao.org/agris-search/search/display. do? $\mathrm{f}=1986 \% 2 \mathrm{FUS} \% 2 \mathrm{FUS} 86221 . \mathrm{xml} \% 3 \mathrm{BUS} 8637294>$. Acesso em: 20 jan. 2013.

MASTRANGELO, T.; CHAUDHURY, M.F.; SKODA, S.R.; WELCH, J.B.; SAGEL, A.; WALDER, J.M.M. Feasibility of using a Caribbean Screwworm for SIT Campaigns in Brazil. Journal of Medical Entomology, Lanham, v.49, p. 1495-1501, 2012. Acesso em: 01 jan. 2013. <http://www.bioone.org/doi/full/10.1603/ ME11273>.

MELVIN, R.; BUSHLAND, R.C. A method of rearing Cochliomyia americana $C$. and $P$. on artificial media. Washington, DC: USDA, Bureau of Entomology and Plant Quarantine, 1936. (Report ET-88). Disponível em: <http://books.google.com.br/ books/about/A_Method_of_Rearing_Cochliomyia_American. $\mathrm{html}$ ?id=mVNnMwEACAAJ\&redir_esc=y>. Acesso em: 20 jan. 2013.

RABÊLO, K.C.; THYSSEN, P.J.; SALGADO, R.L.; ARAÚJO, M.S.; VASCONCELOS, S.D. Bionomics of two forensically important blowfly species Chrysomya megacephala and Chrysomya putoria (Diptera: Calliphoridae) reared on four types of diet. Forensic Science International, Turku, v.210, p.257-262, 2011. Diposnível em: <http://dx.doi.org/10.1016/j. forsciint.2011.03.022. Acesso em: 20 jan. 2013.

SILVA, C.E.; MOYA-BORJA, G.E.; AZAMBUJA, P. Use of polyester pad as a new physical substrate for rearing Cochliomyia hominivorax coquerel(Diptera: Calliphoridae) larvae. Neotropical
Entomology, Londrina, v. 37, p. 349-351. 2008. Diponível em: $<$ http://dx.doi.org/10.1590/S1519-566X2008000300019>. Acesso em: 20 jan. 2013.

STATISTICAL ANALYSIS SYSTEM. SAS Version 9.1. Cary, NC: SAS Institute Inc., 2003.

TAYLOR, D.B. Comparison of two gelling agents for screwworm (Diptera: Calliphoridae) larval diet. Journal of Economic Entomology, College Park, v. 81, p. 1414-1419, 1988. Disponível em: $<\mathrm{http} / /$ digitalcommons.unl.edu/cgi/viewcontent.cgi?article= 1217\&context=entomologyfacpub $>$. Acesso em: 20 jan. 2013.

TAYLOR, D.B.; BRUCE, J.C.; GARCIA, R. Gelled diet for screwworm (Diptera: Calliphoridae) mass production. Journal of Economic Entomology, College Park, v. 84, p. 927-935, 1991. Disponível em: <http://digitalcommons.unl.edu/cgi/viewcontent. cgi? article $=1215 \&$ context $=$ entomologyfacpub $>$. Acesso em: 20 jan. 2013.

TAYLOR, D.B.; MANGAN, R.L. Comparison of gelled and meat diets for rearing screwworm, Cochliomyia hominivorax (Diptera: Calliphoridae) larvae. Journal of Economic Entomology, College Park, v. 80, p. 427-432, 1987. Disponível em: <http:// digitalcommons.unl.edu/cgi/viewcontent.cgi? article $=1218 \&$ conte $\mathrm{xt}=$ entomologyfacpub $>$. Acesso em: 20 jan. 2013.

VARGAS-TERÁN, M.; HOFMANN, H.C.; TWEDDLE, N.E. 2005. Impact of screwworm eradication programmes using the sterile insect technique. In: DYCK, V.A.; HENDRICHS, J.; ROBINSON, A.S. Sterile insect technique: principles and practice in area-wide integrated pest management. Berlin: Springer, 2005. p. 629-650. Disponível em: <http://link.springer.com/chapter/10.1 007\%2F1-4020-4051-2_24>. Acesso em: 20 jan. 2013.

VREYSEN, M.J.B.; GERARDO-ABAYA, J.; CAYOL, J.P. Lessons from area-wide integrated pest management (AW-IPM) programmes with an SIT component: an FAO/IAEA perspective. In: VREYSEN, M.J.B.; ROBINSON, A.S.; HENDRICHS, J. (Org.). Area-wide control of insect pests: from research to field implementation. Dordrecht: Springer, 2007. p. 723-744. Disponível em: <http://www.academia.edu/2922739/AREAWIDE INTEGRATED PEST MANAGEMENT AND THE STERILE_INSECT_TECHNIQUE $>$. Acesso em: 20 jan. 2013. 